the failure to establish reversibility in vitro: the $30 \mathrm{~S}$ subunits, after removal of the factor, cannot be made to reassociate with $50 \mathrm{~S}$ particles, no matter what the magnesium concentration. It must at the same time be recalled that isolated $30 S$ and $50 S$ particles were earlier shown to associate only after formation of the complex between the $30 S$ ribosome, messenger and initiating $t$ RNA. 'The' mechanism of the association of the subunits is still therefore a somewhat clouded issue.

A report by Bonanou et al., however (Biochem. $J$., 110,$87 ; 1968$ ), demonstrates-certainly for the first time in a mammalian system-that conditions exist under which the dissociation is indeed reversible. Dissociation is in this case achieved by exposing the $80 S$ ribosomes to $0.5 \mathrm{M}$ potassium chloride. In this medium the extent of dissociation is concentrationdependent, and evidently follows a true reversible equilibrium. Dialysis against this salt solution yields biologically inactive subunits. These could be dispersed in a magnesium-free buffer, and on addition of magnesium ions ribosomes reappeared and a considerable proportion of biological activity (20-40 per cent) returned. 'This was increased to up to 100 per cent on adding the supernatant from the potassium chloride wash, which would be expected to contain part of the transfer factors.

The recovery of native ribosomes from magnesiumdeficient unfolded states is also a complex matter. In $E$. coli ribosomes various intermediates in the unfolding have been observed, and some of the steps are evidently reversible, though reversibility from the final magnesium-free state, reported early on by one group, was proved illusory. Weller et al. (Biochemistry, 7,$3668 ; 1968$ ) have confirmed that the transitions of the $50 S$ particle to $40 S$, and apparently also the $30 S$ to a partly unfolded state, are reversible, whereas a further step leading to a $29 \mathrm{~S}$ particle from the large subunit is irreversible. It is conjectured that this may result from the loss of the $5 S$ RNA.

An interesting observation on the $50 \mathrm{~S}$ subunit (Hill et al., Biochem. Biophys. Res. Commun., 33, 151; 1968 ) is the presence of a segment of RNA, readily digestible by ribonuclease. The residual particle remains resistant, but the process is accompanied by a large diminution $(7.1$ to $5.5 \mathrm{ml} / \mathrm{g})$ in intrinsic viscosity. The fragment is estimated to have a molecular weight of 30,000 , and has a curious base composition, with 40 per cent of adenine. It is inferred from the viscosity that this RNA forms an exposed "tail" on the $50 \mathrm{~S}$ subunit.

The ribosome may be all things to all men, but most of all to electron microscopists. Three recent reports give three new versions (not, one hastens to add, necessarily incompatible at all points) of its morphology. Nanninga (Proc. US Nat. Acad. Sci., 61, 614; 1968), using shadowing techniques, deduces that the $50 S$ subunit ( $B$. subtilis) has flat surfaces and is approximately an icosahedron. Bruskov and Kiselev (J. Mol. Biol., 37, 367; 1968), using negative staining, see the $50 S$ subunit as a horseshoe, the hollow making a channel down the $70 \mathrm{~S}$ ribosome. On the flat surfaces two rings of "subunits" are described. Worcel et al. (Proc. US Nat. Acad. Sci., 61, 122; 1968), on the other hand, observe a core down the centre of the large subunit, which it is suggested contains RNA. Filaments round the surface have also in the past been affirmed.
Even therefore when the ribosomes are not RNA polymerase, or other mirages, there appears to be enough choice to suit every prejudice.

\section{INSECT PHYSIOLOGY Ecdysone and DNA Puffs}

\section{from our Cell Biology Correspondent}

$\mathrm{IN}_{\mathrm{N}}$ the current issue of Proc. US Nat. Acad. Sci. (61, 971 ; 1968), Crouse reports that the insect moulting hormone, ecdysone, appears to control the formation of DNA puffs in the giant polytene chromosomes of the salivary gland cells in the fly Sciara coprophila. Moulting hormone has long been associated with stimulation of mitosis in insects, but the direct investigation of the effect of the hormone on DNA and RNA synthesis depends, of course, on a supply of pure hormone and this has only recently become available with the chemical characterization and crystallization of ecdysone.

Crouse injected ecdysone into fourth instar larvae of various ages, and found that within 24 hours the salivary gland chromosomes exhibited characteristic DNA puffs and that autoradiographs indicated that DNA synthesis had been initiated along the whole length of the chromosomes. In effect the ecdysone induces a pattern of puffing six to seven days ahead of schedule. Crouse believes that this relationship between ecdysone and chromosome puffing implies that development and metamorphosis cannot occur without puffing.

Last year Goodman, Goidl and Richart (Proc. US Nat. Acad. Sci., 58, 553; 1967) came to exactly the opposite conclusion. They claimed that Sciara larvae could develop normally without the appearance of chromosome puffs, and they challenged the idea that puffing is a particularly significant feature of gene activity. The evidence for these claims came from experiments in which Sciara larvae were fed the vertebrate hormone cortisone, which, though never found in insects, has a similar chemical structure to ecdysone. The larvae apparently developed quite normally without any sign of puffing until the cortisone was withdrawn, whereon normal puffing behaviour was re-established on schedule. In other words, when the cortisone was withdrawn the pattern of puffs that appeared was the same as that in controls at the same stage in development. The inference from this is that any particular pattern of puffs is not determined by the sequence of preceding puffs.

Crouse, however, reports that she has not been able to repeat these observations; she found, in contrast, that larvae fed cortisone do in fact develop puffs, but that the development is delayed by between three and eight days depending on the regimen. Furthermore, simultaneous injection of cortisone with ecdysone failed to block the induction of puffs within 24 hours of injection, but injection with cortisone alone failed to induce puffs even after 48 hours. It seems, therefore, that ecdysone does regulate puff formation and that this process is essential for development. Perhaps the most likely explanation of the diserepancy between the observations of Goodman et al. and Crouse is, as Crouse suggests, that Goodman and colleagues examined larvae which were too young, their development delayed by cortisone. If this is correct, the Sciara system provides a unique opportunity for investigating hormonal control of nucleic acid synthesis. 